\title{
Vestibular Rehabilitation Therapy Improves Perceived Disability Associated With Dizziness Postconcussion
}

\author{
Steven Nagib and Shelley W. Linens
}

\begin{abstract}
Clinical Scenario: Every year, millions of people suffer a concussion. A significant portion of these people experience symptoms lasting longer than 10 days and are diagnosed with postconcussion syndrome. Dizziness is the second most reported symptom associated with a concussion and may be a predictor of prolonged recovery. Clinicians are beginning to incorporate vestibular rehabilitation therapy (VRT) in their postconcussion treatment plan, in order to address the dysfunctional inner ear structures that could be causing this dizziness. Focused Clinical Question: Can VRT help postconcussion syndrome patients experiencing prolonged dizziness by improving their perceived disability? Summary of Key Findings: Three studies were included: 1 randomized control trial, 1 retrospective chart review, and 1 exploratory study. The randomized control trial compared cervical spine therapy alone to cervical spine therapy in conjunction with VRT to obtain medical clearance for sport. The chart review explored VRT as a treatment for reducing dizziness and improving balance and gait dysfunction. The exploratory study implemented VRT in conjunction with light aerobic exercise to improve perceived disability associated with dizziness postconcussion. All 3 studies found statistically significant decreases (improvements) in Dizziness Handicap Index scores. Clinical Bottom Line: There is preliminary evidence suggesting that VRT can improve perceived disability in patients with postconcussion syndrome experiencing prolonged dizziness. There is a decrease (improvement) in Dizziness Handicap Index scores across all 3 studies. VRT is a relatively safe treatment option, with no adverse reactions or case reports. Strength of Recommendation: There is level 2 and level 3 evidence supporting the use of VRT to treat patients suffering from dizziness postconcussion.
\end{abstract}

Keywords: brain concussion, recovery, sport medicine, survey

\section{Clinical Scenario}

Although headache is the most commonly reported symptom associated with concussion, dizziness comes in at a close second with report rates between $66 \%$ and $77 \% .^{1}$ This statistic is concerning as dizziness may be a predictor of prolonged recovery. ${ }^{1}$ Any patient, who has symptoms, including dizziness, persisting greater than 10 days, is diagnosed with postconcussion syndrome (PCS) ${ }^{2}$ Prolonged dizziness can also increase risk of falls, automobile accidents, interrupted workdays, dysfunctional gait, and a delay in return to activity and sport. ${ }^{1}$ Vestibular rehabilitation therapy (VRT) resolves dizziness by targeting structures of the inner ear (peripheral vestibular system) by retraining function and enhancing surrounding structures that can compensate for a dysfunctional vestibular system. There are several outcome measures commonly used to evaluate the efficacy of VRT.

\section{Focused Clinical Question}

Can VRT help patients with postconcussion syndrome experiencing prolonged dizziness improve their perceived disability?

\section{Summary of Search, "Best" Evidence" Appraised, and Key Findings}

- The literature search aimed to find original research exploring any type of VRT implemented after suffering a concussion caused by sport or a direct blow to the head.

The authors are with the Department of Human Physiology, University of Oregon, Eugene, OR. Nagib (snagib@uoregon.edu) is corresponding author.
- Three studies were included: 1 randomized control trial, 1 exploratory study, and 1 retrospective chart review.

- All 3 studies support the use of VRT to treat postconcussion dizziness.

- All 3 studies included used Dizziness Handicap Index (DHI) as one outcome measure.

- The randomized control trial had weekly treatment sessions up to 8-weeks postconcussion. The chart review included a median time between most recent concussion and referral for treatment as 61 days, however, did not mention a timeline for resolution of symptoms. The exploratory study reported improvements at the 3-and 6-month marks. This data indicate that time since concussion is an independent factor of improved perceived disablility. ${ }^{3-5}$

- Further research is needed using a standardized VRT protocol.

\section{Clinical Bottom Line}

There is preliminary evidence showing that VRT can improve perceived disability in patients with PCS experiencing prolonged dizziness. VRT is a relatively safe form of therapy, and no known adverse reactions or cases have been reported. Patient progress with VRT can be tracked via the DHI. The preliminary evidence presented supports active rehabilitation after concussion instead of continued cognitive and physical rest.

\section{Strength of Recommendation}

There is level 2 and level 3 evidence supporting that VRT can reduce dizziness and improve DHI scores in patients with PCS 
because it has historically been used to treat patients with benign paroxysmal positional vertigo. Research exploring VRT as a treatment of PCS is still in its infancy. The goal of this critically appraised topic was to shed light on 1 specific outcome measure that can be explored in future research.

\section{Search Strategy}

\section{Terms Used to Guide Search Strategy}

- Patient/Client Group: patients who suffered a concussion as a result of direct trauma

- Intervention (or Assessment): VRT

- Comparison: no VRT or another type of therapy

- Outcome(s): a decrease (improvement) in DHI score

\section{Sources of Evidence Searched (Databases)}

- PubMed

- Web of Science

- OregonPDF

- SPORTDiscus

- Academic Search Premier

- Search supplemented by cross reference and hand searches

\section{Inclusion and Exclusion Criteria}

\section{Inclusion Criteria}

- Original research

- Human subjects

- Concussion resulting from direct trauma

- Referred for vestibular rehabilitation

- Use DHI as an outcome measure

\section{Exclusion Criteria}

- Non-English

- Abstract only

- Conference proceedings

- Concussions associated with severe traumatic brain injury (ie, open skull fractures)

- Concussions caused by blast injuries (ie, military studies)

\section{Results of Search}

The 3 relevant studies are categorized in Table 1. Level of evidence applied to each study was based on the Oxford Centre for Evidence-Based Medicine 2011 Levels of Evidence. ${ }^{6}$ Table 1 presents the summary of study designs of the articles retrieved.

\section{Best Evidence}

The included studies were identified as the "best" evidence and selected because they met the inclusion criteria without violating any of the exclusion criteria. All 3 studies are clinically relevant and propose a solution for clinicians treating patients suffering from PCS. All included studies are relatively recent-published within last 7 years.
Table 1 Summary of Study Designs of Retrieved Articles

\begin{tabular}{lll}
\hline $\begin{array}{l}\text { Level of } \\
\text { evidence }\end{array}$ & $\begin{array}{l}\text { Study design/methodology } \\
\text { of retrieved articles }\end{array}$ & Author \\
\hline 2 & Randomized control trial & $\begin{array}{l}\text { Schneider } \\
\text { et } \mathrm{al}^{3}\end{array}$ \\
3 & Retrospective chart review & $\begin{array}{l}\mathrm{Alsalaheen}_{\text {et al }}^{4} \\
\text { Soore }^{5} \\
\text { et al }\end{array}$ \\
\hline
\end{tabular}

\section{Summary of Best Evidence}

Table 2 presents the characteristics of included studies.

\section{Implications for Practice, Education, and Future Research}

Active rehabilitation following a concussion is at the center of an emerging field of research and an important topic in contemporary science. The latest consensus statement on concussion management still recommends cognitive rest in the acute phase (first 24-48 h), followed by a graded return to activity. ${ }^{7}$ However, for the first time, the statement includes a section about active concussion rehabilitation. ${ }^{7}$ Unfortunately, it only addresses subthreshold symptom exercise to facilitate recovery, stating that other forms of rehabilitation (including VRT) need further research. VRT aims to promote neural plasticity within existing and relatively intact neural systems. ${ }^{8}$ The 3 main categories of VRT are Habituation, which includes continuous and progressive exposure to symptom-provoking stimuli until those stimuli no longer produce symptoms. ${ }^{8}$ Adaptation involves training nonaffected parts of the peripheral vestibular system to compensate for compromised areas. ${ }^{8}$ Substitution incorporates exercises that use saccade strategies to tract diagonally moving objects, with or without head movement. ${ }^{8}$ Patients who undergo VRT should first have their symptoms (frequency and intensity) under control, as this allows for the most effective treatment. ${ }^{9}$ Similar to any other rehabilitation technique, patients should perform these exercises under the supervision of a trained clinician.

This study focused on the DHI as the main outcome measure used to evaluate the efficacy of VRT, as it is a questionnaire that monitors the status of an individual patient recovering from a concussion. ${ }^{3-5}$ It is a practical, cost-effective, and easy tool to implement across many clinical environments. The DHI is a 25-item questionnaire that assesses the physical, functional, and emotional stress dizziness imposes on a patient's daily life. ${ }^{10}$ It is a valid objective measure, with good internal consistency $(\alpha=.89)$ and high test-retest reliability $(r=.97) .{ }^{11} \mathrm{DHI}$ scores fall on a scale from 0 to 100 , and each question is answered by choosing: yes (4 points), sometimes ( 2 points), and no (0 points). ${ }^{11}$ The questionnaire is easy to implement and interpret results- the lower the score, the less symptomatic is the patient. A decrease of 18 points or more is considered the minimally clinical important difference. ${ }^{4}$ Any clinician working with a patient being treated for dizziness, whether or not the patient has suffered a concussion, can use the DHI. 
Table 2 Characteristics of Included Studies

\begin{tabular}{|c|c|c|}
\hline & Schneider et $\mathrm{al}^{3}$ & Alsalaheen et $\mathrm{al}^{4}$ \\
\hline Study design & Randomized control trial & Retrospective chart review \\
\hline Participants & $\begin{array}{l}31 \text { patients (ages } 12-30 \mathrm{y} ; 18 \text { males, } \\
13 \text { females) } \\
\text { Patients presented to clinic after } \\
\text { suffering an SRC and experiencing } \\
\text { dizziness, neck pain, and/or headache } \\
\text { for } 10+\text { d. If VRT was deemed } \\
\text { appropriate, they were referred to } \\
\text { proper clinicians and included in } \\
\text { this study }\end{array}$ & $\begin{array}{l}114 \text { patients (ages } 8-73 \text { y; } 67 \text { chil- } \\
\text { dren, } 47 \text { adults), who were referred } \\
\text { for VRT following SRC } \\
30 \text { patients did not receive treatment } \\
\text { after initial visit for reasons includ- } \\
\text { ing: VRT not indicated }(n=6) \text {, } \\
\text { distance to clinic }(n=8) \text {, and } \\
\text { noncompliance }(n=16)\end{array}$ \\
\hline $\begin{array}{l}\text { Intervention } \\
\text { investigated }\end{array}$ & $\begin{array}{l}\text { Patients in the intervention group } \\
\text { received customized VRT and } \\
\text { cervical spine therapy. Patients in } \\
\text { the control group only received } \\
\text { cervical spine therapy } \\
\text { Each group underwent weekly } \\
\text { treatment sessions for up to } 8 \mathrm{wk} \text {. } \\
\text { The main outcome measure was } \\
\text { medical clearance for activity; } \\
\text { therefore, there were } 2 \text { populations } \\
\text { within each (treatment and control) }\end{array}$ & $\begin{array}{l}84 \text { out of } 114 \text { patients admitted for } \\
\text { VRT at a specialty clinic received a } \\
\text { customized VRT plan and had at least } \\
1 \text { visit with a trained clinician } \\
\text { VRT plans included: gaze stabiliza- } \\
\text { tion exercises (mainlining a fixed } \\
\text { gaze while turning head from side to } \\
\text { side), balance stabilization activities } \\
\text { with eyes open and closed, walking } \\
\text { with balance challenges, and canalith } \\
\text { repositioning maneuvers }\end{array}$ \\
\hline
\end{tabular}
group

\section{$\mathrm{DHI}^{\mathrm{a}}$}

Patients in the treatment group who were cleared $(n=11)$ had a median change in DHI score of -24 (range: -50 to -6$)$. Those in this group who were not cleared $(n=4)$ had a median change of -13 (range: -16 to -8 ) In the control group, the only patient who was medically cleared had a 48-point decrease in his DHI. Those in this group who did not obtain medical clearance $(n=12)$ had a median score change of -21 (range: -58 to 2 )

Patients who were medically cleared had greater improvements in DHI scores $(P=.02)$

Level of evidence

Validity score

Conclusion
2

9/11 on PEDro scale

VRT is more effective in decreasing DHI scores compared with traditional cervical spine therapy alone, when treating post-SRC patients who have been experiencing symptoms for $>10 \mathrm{~d}$

\section{DHI ${ }^{a}$}

The 30 patients who did not return for a second visit had statistically lower (better) mean DHI scores (37 [19]) at initial evaluation than the 84 who received subsequent treatment (48 [22])

Pretreatment and posttreatment mean DHI scores of the 84 patients who received treatments were 49 (21) and 30 (22), respectively $(P<.001)$

The mean magnitude of change was greater than the MCID established for DHI scores (18 points)

The median length of treatment was 4 visits in $33 \mathrm{~d}$

3

N/A

VRT is effective in clinically decreasing DHI scores in patients with PCS. The median time between most recent concussion and referral for treatment was $61 \mathrm{~d}$, indicating that VRT can be effective for moderate PCS cases

\section{Moore et al $^{5}$}

Exploratory study

14 patients (ages 18-72 y; 6 males, 8 females), who met the World Health Organization's criteria for PCS. All patients suffered concussions by blunt trauma to the head. Patients were also separated into 2 groups $( \pm 18$ y) to determine if age was a confounding factor

All patients completed a supervised VRT program and prescribed subsymptom threshold aerobic exercise. The VRT was a home-based program with visits to the office at different time points to review exercises and modify as needed. Frequency of these follow-up visits depended on the decision of the patient with his/her clinician

9 patients also received undisclosed treatment at other clinics including orthopedic physical therapy, occupational therapy, visual therapy, and behavioral therapy

$\mathrm{DHI}^{\mathrm{a}}$

Mean DHI scores were 58.1 (17.8) at initial evaluation, 48.2 (15.7) at 3-mo follow-up, and 32 (20.9) at 6-mo follow-up

There were statistically significant decreases in DHI scores between initial evaluation and 3-mo follow-up and initial evaluation and 6-mo follow-up $(P<.001)$, but not between 3-mo and 6-mo follow-up

3

N/A

DHI scores improved within the first 3 mo of VRT and kept improving in patients who continued treatment. There was no significant difference in outcome based on age of patient

\footnotetext{
Abbreviations: DHI, Dizziness Handicap Index; MCID, minimal clinically important difference; N/A, not applicable; PCS, postconcussion syndrome; PEDro, Physiotherapy Evidence Database; SRC, sports-related concussion; VRT, vestibular rehabilitation therapy.

${ }^{a}$ There were multiple outcome measures in each study; however, for the purposes of this critically appraised topic, only the information surrounding DHI was extracted, reviewed, and included in this table.
}

The 3 studies included in this paper found statistically significant improved DHI scores following VRT. In the randomized control trial by Schneider et $a l,{ }^{3}$ the authors used medical clearance as the main outcome. Patients who were medically cleared had greater improvements in DHI scores $(P=.02)$. Of these patients,
11 had a median decrease in DHI score of 24. In the retrospective review by Alsalaheen et al, ${ }^{4}$ pretreatment and posttreatment mean DHI scores of the 84 patients who received treatments were 49.0 (21.0) and $30.0(22.0)$, respectively $(P<.001)$. The mean magnitude of decrease was greater than the minimally clinical important 
difference established for DHI scores. In the exploratory study by Moore et al, ${ }^{5}$ mean DHI scores were 58.1 (17.8) at initial evaluation and 32.0 (20.9) at 6-month follow-up, which was a statistically significant decrease in DHI scores between initial evaluation and 6-month follow-up $(P<.001)$. To date, this critically appraised topic is the first of its kind to assess the efficacy of VRT as validated by an accepted outcome measure, the DHI; thus, there are many elements of VRT that need to be addressed.

Concerning timing of treatment, Alsalaheen et $\mathrm{al}^{4}$ reported the median time between most recent concussion and referral for treatment as 61 days, indicating that VRT can be effective for chronic PCS cases. ${ }^{4}$ However, that study also recognized that regardless of how long a patient received treatment, the critical factor was the number of treatment sessions. More treatment sessions in a shorter amount of time produced similar outcomes as the same amount of treatments over a longer period. ${ }^{4}$ Schneider et $\mathrm{al}^{3}$ suggested the opposite, as all the participants in that study were on the same treatment schedule, and at some point over an 8-week period each individuals' symptoms resolved. The findings of Moore et $\mathrm{al}^{5}$ cannot be directly compared with the other 2 studies, as that group collected data at fixed points over time (initial evaluation, 3-mo, and 6-mo postinjury). Frequency of and adequate number of treatments need to be addressed by research, especially as insurance companies are increasingly limiting patient-therapist interaction. The authors of the 3 studies did not suggest a recommended frequency or number of treatment sessions; however, based on the timelines of each study, it seems that most symptoms will resolve from 6 to 12 weeks after initial treatment. Nonetheless, each study showed significant improvements in DHI scores after VRT. Although it is clear that perceived disability improved, there may have been another improvement that was not measured-gait.

High DHI scores are correlated with ongoing gait disturbances. ${ }^{12}$ Gait impairments can be present more than 2-months postconcussion, well after the majority of athletes return to play. ${ }^{13}$ Motor control deficiency following concussion is a significant concern, as it has been correlated with increased risk of injury. ${ }^{14}$ If gait analysis becomes an accepted objective and measurable concussion diagnostic tool, researchers should continue to explore the correlation between VRT, DHI, and gait. If VRT can normalize disrupted gait and improved gait is correlated with improved DHI scores, then VRT will be further validated and popularized as a form of concussion treatment.

There are many variables and confounding factors further research needs to uncover. As mentioned previously, more studies need to address the timing at which VRT is initiated postconcussion and the frequency at which treatment is received. Continuing to correlate factors such as gait and perceived disability will integrate multiple systems of the human body for a more holistic approach. In addition, gearing questions toward specific populations can eliminate discrepancy in diagnoses and treatment effect. A difficult part of managing concussions is the sometimes vagueness in presentation, or rather no presentation, of a wide array of symptoms with varying intensity in individual patients. The vestibular-ocular reflex, responsible for stabilizing gaze as the head moves, integrates information from the ocular and vestibular systems. ${ }^{1}$ Thus, a presence of visual changes following a concussion should be taken into consideration when referring for VRT and continuously evaluated after VRT sessions to track improvements. ${ }^{1}$ This also makes standardization of a VRT protocol difficult. Although VRT, like any treatment, should be personalized to the patient, future research needs to include exactly what is being done during the clinical intervention so that comparison between studies can be accurate and valid. Once trends are uncovered, there may be a possibility for a standard protocol.

As with any treatment-based study, there is always going to be some expectation by a participant that he/she will have improved symptoms after a treatment session. Future research should continuously track improvements via multiple tests and questionnaires to continue to uncover and create the best management plan for concussed patients. Finally, a long-term followup session should be conducted months to 1 year after being discharged from treatment to ensure symptoms have not returned. Clinicians should incorporate VRT when treating concussed patients experiencing prolonged dizziness. By presenting an alternative to cognitive and physical rest, this study adds to a plethora of concussion-based research and warrants more research to be done on VRT.

\section{References}

1. Valovich McLeod TC, Hale TD. Vestibular and balance issues following sport-related concussion. Brain Inj. 2015;29(2):175-184. PubMed ID: 25291297 doi:10.3109/02699052.2014.965206

2. Leddy JJ, Baker JG, Willer B. Active rehabilitation of concussion and post-concussion syndrome. Phys Med Rehabil Clin N Am. 2016;27:437-454. PubMed ID: 27154855 doi:10.1016/j.pmr.2015. 12.003

3. Schneider KJ, Meeuwisse WH, Nettel-Aguirre A, et al. Cervicovestibular rehabilitation in sport-related concussion: a randomised controlled trial. Br J Sports Med. 2014;48(17):1294-1298. PubMed ID: 24855132 doi:10.1136/bjsports-2013-093267

4. Alsalaheen BA, Mucha A, Morris LO, et al. Vestibular rehabilitation for dizziness and balance disorders after concussion. J Neurol Phys Ther. 2010;34(2):87-93. PubMed ID: 20588094 doi:10.1097/NPT. 0b013e3181dde568

5. Moore BM, Adams JT, Barakatt E. Outcomes following a vestibular rehabilitation and aerobic training program to address persistent post-concussion symptoms an exploratory study. J Allied Health. 2016;45(4):e59-e68. PubMed ID: 27915363

6. Howick J, Chalmers I, Glasziou P, et al. The 2011 Oxford CEBM Evidence Levels of Evidence (Introductory Document). Oxford Centre for Evidence-Based Medicine. 2011:1-3.

7. McCrory P, Meeuwisse W, Dvořák J, et al. Consensus statement on concussion in sport- the 5th international conference on concussion in sport held in Berlin, October 2016. Br J Sports Med. 2017; 51(11):838-847. PubMed ID: 28446457 doi:10.1136/bjsports2017-097699

8. Aligene K, Lin E. Vestibular and balance treatment of the concussed athlete. NeuroRehabilitation. 2013;32(3):543-553. PubMed ID: 23648608 doi:10.3233/NRE-130876

9. Gurley JM, Hujsak BD, Kelly JL. Vestibular rehabilitation following mild traumatic brain injury. NeuroRehabilitation. 2013;32(3):519528. PubMed ID: 23648606 doi:10.3233/NRE-130874

10. Treleaven J. Dizziness handicap inventory (DHI). Aust J Physiother. 2006;52(1):67. PubMed ID: 16555410 doi:10.1016/S0004-9514(06) 70070-8

11. Jacobson G, Newman C. The development of the Dizziness Handicap Inventory. Arch Otolaryngol Head Neck Surg. 1990;116(4):424-427. PubMed ID: 2317323

12. Zanotto D, Mamuyac EM, Chambers AR, et al. Dizziness Handicap Inventory score is highly correlated with markers of gait disturbance. 
Otol Neurotol. 2017;38(10):1490-1499. PubMed ID: 28984811 doi:10.1097/MAO.0000000000001586

13. Howell DR, Osternig LR, Chou LS. Dual-task effect on gait balance control in adolescents with concussion. Arch Phys Med Rehabil. 2013;94(8):1513-1520. PubMed ID: 23643687 doi:10.1016/j.apmr. 2013.04.015
14. Brooks MA, Peterson K, Biese K, Sanfilippo J, Heiderscheit BC, Bell DR. Concussion increases odds of sustaining a lower extremity musculoskeletal injury after return to play among collegiate athletes. Am J Sports Med. 2016;44(3):742-747. PubMed ID: 26786903 doi: $10.1177 / 0363546515622387$ 